\author{
Richard A. Miller \\ Gonzalo Garcia \\ Christopher J. Kirk \\ jacek M. Witkowski
}

\section{Early activation defects in \\ $T$ lymphocytes from aged mice}

Authors' addresses

Richard A. Miller', Gonzalo Garcia2,

Christopher J. Kirk ${ }^{3}$, Jacek M. Witkowski+,

'Department of Pathology, Geriatrics Center,

Institute of Gerontology, and Ann Arbor DVA

Medical Center, University of Michigan,

Ann Arbor, Michigan, USA.

2Department of Pathology, University of

Michigan, Ann Arbor, Michigan, USA.

${ }^{3}$ Graduate Program in Molecular and Cellular Biology, University of Michigan, Ann Arbor, Michigan, USA.

${ }^{4}$ Medical Academy of Gdansk, Gdansk, Poland.

\section{Correspondence to:}

Richard A. Miller

University of Michigan Geriatrics Center

CCGCB Room 5316

Box 0940

1500 East Medical Center Drive

Ann Arbor MI 48109-0940

USA

Fax: 13139369220

e-mail: millerr@umich.edu

\section{Acknowledgements}

The work described here was supported largely by NIA grant AG09801, and to a smaller extent by AG08808 and AG03978. CJK was supported by training grants AI07413 and GM07315, and by an AFAR/Glenn summer fellowship.

Immunological Reviews 1997

Vol. 160: 79-90

Printed in Denmark. All rights reserved

Copyright $\mathcal{O}$ Munksgaard 1997

Immunological Reviews

ISSN 0105-2896
Summary: Aging affects both calcium signals and protein kinase cascades in mouse $\mathrm{T}$ lymphocytes. The decline in calcium signal development largely represents differences between naive and memory $\mathrm{T}$ cells; the latter are resistant to increases in calcium concentration, and are more common in aged mice. Aging leads to declines in phosphorylation of a wide range of substrates in T cells stimulated by either anti-CD3 antibodies or by substances, such as phorbol myristate acetate (PMA) or ionomycin, that act at intracellular sites, but some phosphoproteins respond only in old T cells, and others respond regardless of age. Tyrosine phosphorylation of the $\mathrm{CD} 3 \zeta$ chain declines with age, both in resting $\mathrm{T}$ cells and after activation, but the proportion of Zap-70 that is bound to $\mathrm{CD} 3 \zeta$ increases in $\mathrm{T}$ cells from old mice. Zap-70 function and phosphorylation of $\mathrm{CD} 3 \zeta$-associated Zap-70 change only slightly after stimulation of $\mathrm{T}$ cells by anti-CD3 and anti-CD4, and are at similar levels in activated old and young T cells. Nonetheless, induction of Raf-1, MEK, and ERK kinase activity declines with age in CD4 T cells. The effect of aging on T-cell activation is not simply an overall decline in signal intensity, but a set of qualitative changes that differ among subsets and depend at least partly on the nature of the stimulus.

\section{Introduction}

Mitogenic agents, including antibodies to the TCR and CD3 complex, induce in resting $\mathrm{T}$ cells a complex chain of events, including an increase in intracellular calcium ion concentration and activation of a cascade of protein kinase signals, that lead to new gene transcription within $15 \mathrm{~min}$ and eventually bring about cytokine production and cell division. T cells from aged mice (which for brevity's sake will often be referred to in this paper as "old T cells" to distinguish them from "young" $T$ cells, i.e. cells derived from a young donor) exhibit defects in this activation process within the first $60 \mathrm{~s}$ after exposure to a stimulus. The research challenge is to determine which of these defects if any - contribute to the decline with age in protective immunity, i.e. the poor performance of the aged immune system when confronted with an infectious or neoplastic challenge.

This task is complicated by a number of technical factors. For one thing, the chain of events that mediate activation of the normal T cell (i.e. T cells from young donors) is still far from clear; although current consensus models $(1,2)$ are far more 
detailed than their ancestors of two to ten years ago, they still contain many redundancies, missing links and ill-defined feedback loops. A second problem is that most of our current knowledge of T-cell activation has been gained by studies of relatively tractable lymphoma lines and other varieties of transformed cell whose signal transduction pathways turn out to resemble those of normal $\mathrm{T}$ cells, freshly isolated from actual donors, in some but by no means all respects.

The heterogeneity of a freshly isolated T-cell preparation poses a third kind of technical obstacle: such a preparation consists of a mixture of many different cell types - among which CD4 naive, CD4 memory, CD8 naive, and CD8 memory are the most salient - whose activation pathways and responses to aging may be quite distinct. This heterogeneity creates a number of chances for confusion, since age-dependent changes might affect only one subset (and thus be missed by tests of unseparated $\mathrm{T}$ cells), but also because the relative proportions of these cell subsets change systematically with age, so that ageinvariant differences between subsets can create the impression of an age effect on signal development itself. Studies of intracytoplasmic free calcium ion concentration $\left([\mathrm{Ca}]_{i}\right)$ provide a useful example. Memory $\mathrm{T}$ cells, from mice of any age, produce lower levels of $[\mathrm{Ca}]_{\mathrm{i}}$ in responses to mitogenic lectins, anti-CD3 antibodies, or calcium ionophores $(3,4)$, and thus the relative increase with age in memory $\mathrm{T}$ cells leads to an overall decline in mitogen-induced calcium signals when tested on T cells not separated into naive and memory subsets $(3,5)$.

Expense is a fourth obstacle to progress on aging and T-cell activation. To produce $10^{9}$ Jurkat cells costs about $\$ 50$ in materials. To produce $10^{9}$ splenic T cells from 24-month old mice costs well over $\$ 2,000$ for the mice alone (at NIA-subsidized prices; 4-fold higher otherwise), assuming 100\% cell yield and no attrition due to ill health of the mice. To produce $10^{9} \mathrm{CD} 4$ memory cells from 24-month old mice costs $\$ 6,000$ for the mice alone. These economic constraints put a premium on microassay methods, and preclude some kinds of biochemical analysis altogether.

Nonetheless, some insights into the ways in which aging alters the early events in T-cell activation have begun to emerge, and this essay will briefly present some of the earlier lessons as a prelude to illustration of more recent work and discussion of questions for future investigation.

\section{Calcium signals}

Work from our laboratory on age-related changes in calcium signal development has been reviewed elsewhere $(5,6)$, and will be summarized only briefly here. Using indo- 1 to monitor $[\mathrm{Ca}]_{i}$, we found that $\mathrm{T}$ cells from old mice generated lower average $[\mathrm{Ca}]_{i}$ levels after exposure to concanavalin A (Con A) than $\mathrm{T}$ cells from younger donors, and that this difference reflected a decline in the proportion of $\mathrm{T}$ cells that could generate a calcium signal after activation (7). Using flow cytometry to measure $[\mathrm{Ca}]_{\mathrm{i}}$ in individual cells with specific surface marker phenotypes showed that the decline with age in the proportion of responsive $\mathrm{T}$ cells represented the gradual replacement of naive (i.e. CD 44 $4^{\mathrm{lo}}$ ) T cells by memory (CD44 ${ }^{\mathrm{hi}}$ ) cells, which generated lower $[\mathrm{Ca}]_{\mathrm{i}}$ signals in response to mitogenic lectins at any age (3). The differences between young and old cells, and the corresponding differences between naive and memory cells, could not be explained entirely by changes in receptor-dependent signal pathways (including for example protein kinase-dependent activation), because differences in $[\mathrm{Ca}]_{\mathrm{i}}$ were also observed when the old $\mathrm{T}$ cells, or memory $\mathrm{T}$ cells from mice of any age, were exposed to the calcium ionophore ionomycin, which bypasses receptor-dependent signals by allowing calcium to flow into the cell along the $10^{4}$-fold transmembrane concentration gradient $(3,8)$. Differences between young and old T cells (9), and between naive and memory cells (4) in resistance to ionomycin-induced changes in $[\mathrm{Ca}]_{\mathrm{i}}$ have also been reported by other laboratories.

The biochemical mechanism(s) that underlie these effects of age and subset are not entirely clear. We do not believe that the age defect in calcium signal generation reflects a decline in IP3 production, in part because we find no effect of age on IP3 production (10) and in part because in freshly isolated T cells (unlike lymphoma model systems) the contribution of IP3mediated release of calcium from pre-existing internal stores is slight and transient (11), accounting in our own experience to only about $15 \%$ of the total calcium signal in the first minute, and less thereafter. The resistance of memory $\mathrm{T}$ cells (and therefore $\mathrm{T}$ cells from old mice) to ionomycin-mediated changes in $[\mathrm{Ca}]_{\mathrm{i}}$ suggests a stronger ability to buffer rapid changes in internal calcium concentration however these are produced, perhaps by increased function or higher sensitivity of the plasma membrane calcium pump.

Several lines of evidence indicate that this resistance to changes in $[\mathrm{Ca}]_{i}$ contributes to defective T-cell function in old age. $\mathrm{T}$ cells, from young or old donors, that fail to generate a calcium signal in response to Con A, anti-CD3, or ionomycin can be isolated by fluorescence-based cell sorting; such cells are found in limiting dilution assays to be largely unable to produce IL-2, or to proliferate or generate cytotoxic effectors in response to Con A and exogenous IL-2 (12). Changes in buoyant density in a Percoll/ionomycin gradient can also be used to 
Fig. 1. Calcium signals in CD4 memory cells of young and old mice: subsets that differ in expression of P-glycoprotein. CD4 memory $\mathrm{T}$ cells were purified by depletion of $\mathrm{CD} 8^{+}$and CD45RB ${ }^{\text {tw }}$ cells, then stained with Rhodamine123 (as a P-glycoprotein indicator) and indo-1 (as a calcium indicator), and then stimulated with either anti-CD3 (left panel) or the calcium ionophore ionomycin (right panel). The bars depict the peak response as changes in $[\mathrm{Ca}]_{i}$ (nM) over the resting baseline levels for one of three replicate experiments. (J.M. Witkowski \& R.A. Miller, unpublished data.)
Anti-CD3

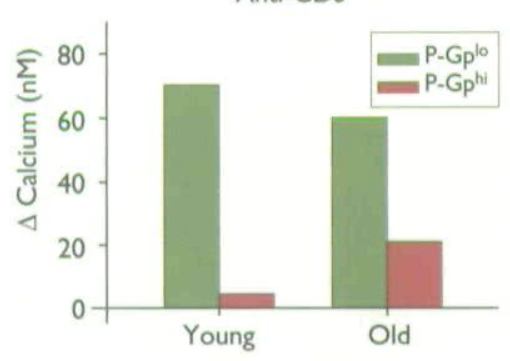

lonomycin

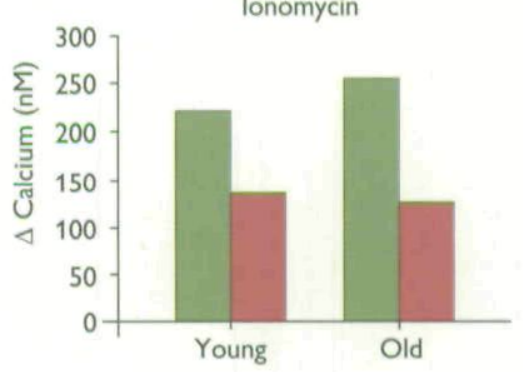

enrich for T cells whose levels of [Ca $]_{i}$ remain stable under conditions of increased calcium influx (13); these calcium buffered cells show the surface markers of memory T cells and, like memory cells isolated by other means, show poor reactivity in tests of IL-2 production and IL-2 responsiveness after stimulation by Con $\mathrm{A}$ or the superantigen staphylococcal enterotoxin $\mathrm{B}$.

These studies show that the effect of age on calcium signals induced by polyclonal activators can largely be accounted for by differences between naive and memory $\mathrm{T}$ cells, and the increase with age in the latter cell subset. They do not, however, provide insights into the molecular basis for age-related declines in memory T-cell function which are manifest, for example, in the poor responses of elderly individuals to recall antigens in skin delayed-type hypersensitivity tests. The CD4 memory population of old mice contains a subset of cells, marked by the expression of the plasma membrane pump Pglycoprotein (P-Gp), which are much less common among CD4 memory cells of young mice (14). P-Gp ${ }^{\text {hi }}$ CD 4 memory T cells from young mice are defective, compared to $\mathrm{P}-\mathrm{Gp}^{\mathrm{lc}}$ cells, in tests for proliferation and production of IL-4, IL-5, IL-10, and IFN- $\gamma$ when stimulated by anti-CD 3 antibodies, and their poor responsiveness cannot be overcome by co-stimuli including $\mathrm{IL}-2$, IL-4, anti-CD28, or phorbol myristate acetate (PMA) (15). Proliferation and IFN- $\gamma$ production by the $\mathrm{P}-\mathrm{GP}^{\mathrm{h}}$ CD4 memory subset can, however, be restored by the combination of PMA and ionomycin, suggesting that defects in $\mathrm{CD} 3$-triggered calcium signal generation might contribute to the apparent anergy of this subset. To test this idea, we have measured $[\mathrm{Ca}]_{\mathrm{i}}$ levels in $\mathrm{P}-\mathrm{Gp}^{\mathrm{hi}}$ and $\mathrm{P}-\mathrm{Gp}^{\mathrm{lo}} \mathrm{CD} 4$ memory cells after stimulation by anti-CD3 and by ionomycin. The results of a representative experiment are shown in Fig. 1 (J.M. Witkowski \& R.A. Miller, unpublished data). P-Gp ${ }^{\text {hi }}$ CD4 memory cells, from old or young mice, do indeed generate relatively poor calcium signals in response to anti-CD3 stimulation. Their relative resistance to ionomycin suggests that the defect in calcium signal generation may reflect abnormally high levels of calcium extrusion or calcium sequestration, and probably cannot be explained simply on the basis of poor signal transduction through the TCR/CD3 complex. Functional responses of CD4 naive $\mathrm{T}$ cells have been shown to decline with age in transgenic mice bearing $\mathrm{T}$-cell receptors specific for pigeon cytochrome $\mathrm{C}$ (16), and it will be interesting to see if this decline represents the relative hyporesponsiveness - and perhaps calcium resistance - of P-Gp $\mathrm{p}^{\text {hi }} \mathrm{T}$ cells.

\section{Protein kinase defects: early studies}

Two-dimensional protein electrophoresis can be used to look for age-dependent changes in the patterns of protein phosphorylation induced by mitogenic agents. The disadvantage of this method is that it provides no information about the identity of the substrates, or the kinases and phosphatases that regulate their phosphorylation. The advantage is that the survey approach, by monitoring many substrates simultaneously, can rapidly provide an overview of patterns in responses to different agonists, a picture that would emerge only very slowly by studies of single substrates. Taking pains to adjust for differences in the specific activity of the cellular ATP pool, and using reference phosphoprotein spots for standardization, can give quantitative estimates of protein phosphate levels whose coefficients of variation across experiments are typically less than $10 \%(17)$.

The pattern of protein phosphorylation induced by a panel of receptor-dependent and receptor-independent activators differed in interesting ways between young and old mice (18). A synopsis of the results is shown in Fig. 2. Panels on the top row show phosphoproteins that responded vigorously, within 10 min of activation, in T cells from young mice, but not at all in T cells from old donors. Some, such as 6559 and five others, responded in young $\mathrm{T}$ cells about equally well to anti-CD3 and to intracellular mediators like PMA and ionomycin. Some, like protein 5952 and three others, responded to anti-CD3 and ion- 

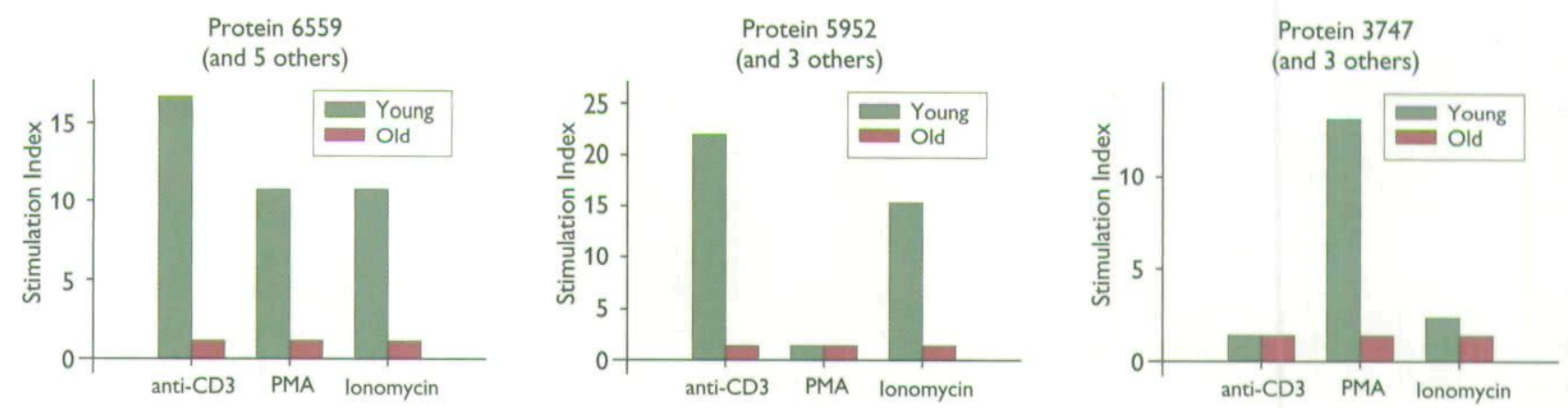

Protein 2955
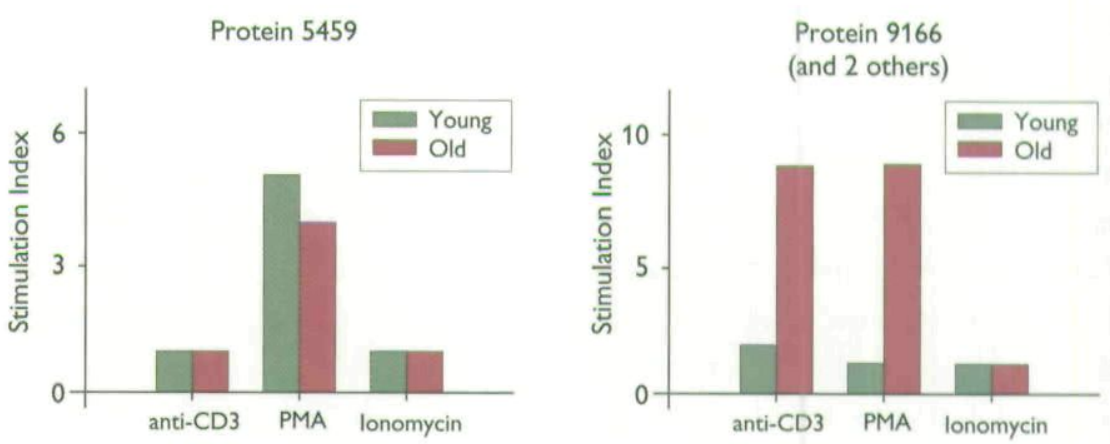

Fig. 2. Analysis of protein phosphorylation patterns using twodimensional gel electrophoresis: synopsis of results. Stimulation indices (activated/resting) for ${ }^{32} \mathrm{P}$ levels in six proteins whose level of phosphorylation changed by at least 5 -fold within 10 min of activation by anti-CD3, PMA, and/or ionomycin in T cells from old or young mice.

omycin, but not to PMA; phosphorylation of these substrates is likely to reflect an increase in $[\mathrm{Ca}]_{\mathrm{i}}$, and not to require protein kinase C (PK-C) activation. A third set, like protein 3747 and three others, showed responses to PMA that were not induced by anti-CD3 or by ionomycin; these are likely to reflect PK-Cdependent pathways that are, for unknown reasons, inhibited by calcium signals (not shown). None of these 14 phosphoproteins responded, in old T cells, to anti-CD3, PMA, or ionomycin. These data suggest that some protein kinase-dependent signals are greatly diminished in $\mathrm{T}$ cells from old mice, and that the defects include alterations in kinase pathways triggered by $[\mathrm{Ca}]_{\mathrm{i}}$ and by PK-C. The published results (18) also show that the effects of aging are gradual, with significant declines seen in mice as young as 10-12 months of age, with progressively larger defects at 17 and 23 months. Since some of the age-sensitive substrates are phosphorylated only in CD4 memory cells, and others only in CD4 naive cells, the differences between young and old $\mathrm{T}$ cells cannot represent merely the replacement of naive by memory cells.
The bars show means of 3 independent experiments; standard errors are not shown, but were almost always less than $10 \%$ of the mean. The protein designations indicate apparent $\mathrm{MW}$ and $\mathrm{pI}$; protein 6559 , for example, has an apparent MW of $65 \mathrm{kDa}$ and a pI of 5.9. From (18).

The bottom panels in Fig. 2 show that the pattern of protein phosphorylation induced in old T cells by these agonists is not simply diminished but instead shows qualitative alterations. Phosphoproteins 2955 and 5459, for example, respond to PMA just as strongly in old T cells as in young T cells. Similarly, protein 9166 and two other, probably related, proteins respond to anti-CD3 and to PMA only in T cells from aged donors (Fig. 2, bottom right panel). Thus the decline in most of the PMA-triggered phosphorylation reactions (see top panel) cannot simply reflect a downregulation of PK-C function, but may instead represent a shift, with age, in the localization of one or more PK-C isoenzymes, in accessibility of PK-C substrates, or in specific phosphatases that reverse PK-C-catalyzed phosphorylations. There is some corollary evidence to suggest a strong effect of age on the localization of distinct PK-C isoforms in resting and mitogen-activated human T cells (19). It may be particularly informative to examine the effects of aging on the localization and activity of the PK-C $\theta$ isoenzyme, thought to play an important role in the commitment of $\mathrm{T}$ cells to activation and new gene expression (20). 
These studies of incorporation of ${ }^{32} \mathrm{P}$ from ATP into phosphoproteins provide information mostly on the function of serine- and threonine-specific protein kinases, since the large majority ( $>99 \%$ ) of protein phosphate groups are added to these two amino acids. The tyrosine-specific protein kinases, however, including fyn, lck, and Zap-70, are known to play a critical role in the T-cell activation process. An early study using antiphosphotyrosine (anti-PY) immunoblot methods quantitated levels of phosphotyrosine (PY) on mitogen-sensitive substrates separated by one-dimensional electrophoresis (21). Three prominent phosphoproteins, with MWs of 40, 80, and $120 \mathrm{kDa}$, were found to be tyrosine-phosphorylated within 2-10 min after stimulation of young $\mathrm{T}$ cells by anti-CD3 or Con $\mathrm{A}$, and the 80 and $120 \mathrm{kDa}$ substrates also responded to anti-TCR antibodies. Each of these reactions was diminished about 2-fold in $\mathrm{T}$ cells from older mice. The shift from naive to memory $\mathrm{T}$ cells appeared to account for some of this decline, in that phosphorylation of all three substrates was weaker (at any age) in CD4 memory cells than in the unseparated CD4 pool (22). These data suggested that aging might impair the activation of tyrosine-specific protein kinases, and set the stage for more detailed analysis of specific kinases and their substrates.

\section{Signal initiation: phosphorylation of $\mathrm{CD} 3 \zeta$ isoforms}

Investigations of the responses of transformed T-cell lines, such as Jurkat, had led to the development of models in which T-cell activation was initiated by dual phosphorylation of one or more of the ITAM regions on the zeta $(\zeta)$ chain of the CD3/TCR receptor complex by the src-family, tyrosine-specific kinases fyn and/or lck. Phosphorylated $\mathrm{CD} 3 \zeta$ then was said to serve as a docking site for the syk-family tyrosine kinase Zap-70. Once Zap-70 was attached to $\mathrm{CD} 3 \zeta$, it was said to be itself tyrosinephosphorylated, either by other Zap-70 molecules or by a srcfamily kinase. Once activated by tyrosine-specific phosphorylation, Zap-70, perhaps together with fyn and/or lck, could catalyze the phosphorylation and activation of a wide range of other signal transducing protein substrates (23). In contrast, studies of normal (i.e. non-transformed) lymphocytes had shown that the $\mathrm{CD} 3 \zeta$ chain was already tyrosine-phosphorylated and associated with Zap-70 in resting peripheral T cells, casting some uncertainty on the idea that phosphorylation of $\mathrm{CD} 3 \zeta$ was itself the key trigger of the activation process (24).

To examine the effects of aging on $\mathrm{CD} 3 \zeta$ phosphorylation, we employed an assay in which anti- $\zeta$ immunoprecipitates from $\mathrm{CD} 4 \mathrm{~T}$ cells were resolved by electrophoresis and then detected by immunoblotting with anti-PY antibody (25). Nonreducing gels were used to separate the various isoforms of the $\zeta \zeta$ dimer, since work in another laboratory had shown a complex pattern of differentially phosphorylated dimers that were poorly resolved after reduction of interchain disulfide bonds (26). This approach led to several results of interest, illustrated in Fig. 3. In agreement with previous published work, we observed high levels of phosphorylated $\zeta \zeta$ dimers even in resting $\mathrm{T}$ cells, i.e. prior to the addition of any mitogenic agent; these are indicated by the ZD 1 arrow in Fig. 3. The level of phosphorylation of the ZD1 isoform was 3- to 6-fold lower in old CD4 cells than in young cells; this difference was statistically significant $(\mathrm{p}<0.001)$ in a series of experiments on 10 mice in each age group. Phosphorylation of the ZD1 band was not altered by T-cell activation using cross-linked anti-CD3 and anti-CD4 antibodies, but remained at constant levels for at least $10 \mathrm{~min}$ after activation (25). The kinase(s) that mediate base-

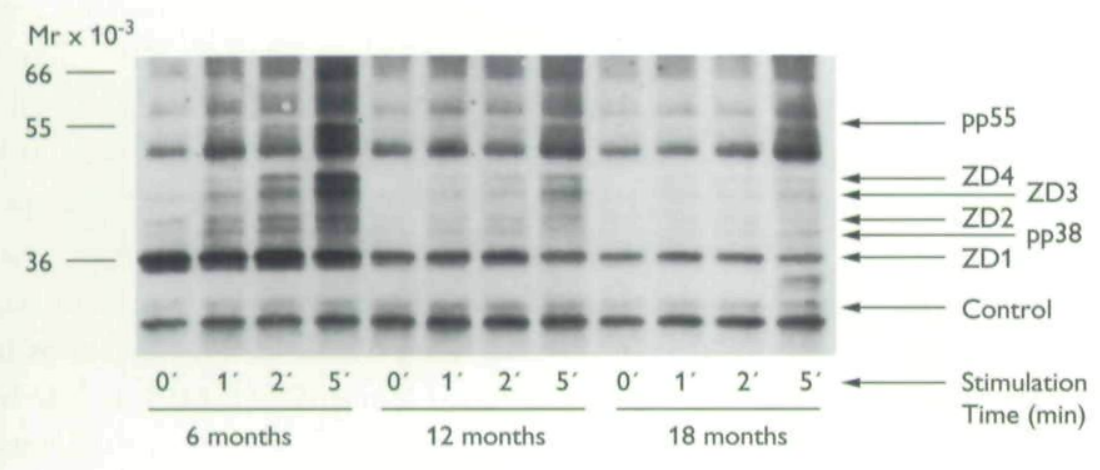

Fig. 3. Age-dependent changes in tyrosine phosphorylation of $\mathrm{CD} 3 \zeta$ chain dimers in resting and activated CD4 $\mathrm{T}$ cells. Cells were stimulated for the indicated times with anti-CD3 and anti-CD4 cross-linked by goat anti-rat IgG. Anti- $\mathrm{CD} 3 \zeta$ immunoprecipitates were separated by electrophoresis on $12 \%$ SDS-PAGE under nonreducing conditions, transferred to nitrocellulose, and detected with antiphosphotyrosine antibody using a chemoluminescence technique. Arrows at the right indicate the positions of four dimers of the $\mathrm{CD} 3 \zeta$ chain (ZD1, ZD2, ZD3, and ZD4), as well as two other $\mathrm{CD} 3 \zeta$-associated phosphoproteins (p38 and p55) whose phosphorylation is stimulated by anti-CD3/CD4 cross-linking, and also an invariant control band used for interassay normalization. 
line phosphorylation of $\mathrm{CD} 3 \zeta$ are not known at present, although it seems likely that both lck and perhaps also other kinases contribute to this process (27). Nor is it clear which tyrosine-specific protein phosphatases might play a role in regulating $\mathrm{CD} 3 \zeta$ phosphorylation in resting $\mathrm{T}$ cells. Our data suggest that $\mathrm{T}$ cells from aged mice are likely to have either lower levels of the responsible kinase(s), higher levels of the phosphatase(s) involved, or altered accessibility of the TCR/CD3 complex.

Fig. 3 also shows the effects of T-cell activation induced by cross-linking surface CD3 and CD4 molecules. Young CD4 cells respond to this stimulus by increased phosphorylation of three other isoforms of $\zeta \zeta$ dimer (bands ZD2, ZD3, and ZD4 in Fig. 3) which migrate more slowly than ZD1 in this non-reducing polyacrylamide gel. Two other $\mathrm{CD} 3 \zeta$-associated bands at apparent MW $38 \mathrm{kDa}$ and $55 \mathrm{kDa}$, shown by diagonal electrophoresis not to be $\zeta$-isoforms (25), were also tyrosine phosphorylated in response to stimulation. Phosphorylation of each of these substrates was apparent within $60 \mathrm{~s}$ (Fig. 3), reached a peak 5 to $10 \mathrm{~min}$ later, and then declined by 20-30 min (25). Phosphorylation of each of these five substrates was much weaker (3- to 4 -fold lower at the 5 min time point; $\mathrm{p}<0.05)$ in CD4 $\mathrm{T}$ cells from old mice. It is not yet clear which enzymes are responsible for phosphorylation and dephosphorylation of these $\zeta \zeta$ isoforms, nor whether they play specific roles (either positive or negative) in the T-cell activation process.

To see whether these effects of age reflected differences between naive and memory $\mathrm{T}$ cell subsets, whose relative proportions shift dramatically with age (28), we carried out a series of experiments, of the sort shown in Fig. 4, testing naive and memory CD4 cells from young and old donors for $\mathrm{CD} 3 \zeta$ phosphorylation levels. The effect of age on ZD-1 phosphorylation, seen in resting CD4 cells, seemed to affect both naive and memory cells to an equal extent. An analysis of variance, with post hoc testing by the Student-Newman-Keuls procedure, found no differences between naive and memory cell ZD-1 phosphorylation at either age. Aging did, however, diminish the extent of ZD-1 phosphorylation in both the naive ( $\mathrm{p}=$ $0.02)$ and memory $(p=0.04)$ subsets; the average effect is about 6-fold, although this is not obvious in the top panel of Fig. 4, which was over-exposed to bring out the more slowly migrating bands. The ZD-2 data, however, show effects of both
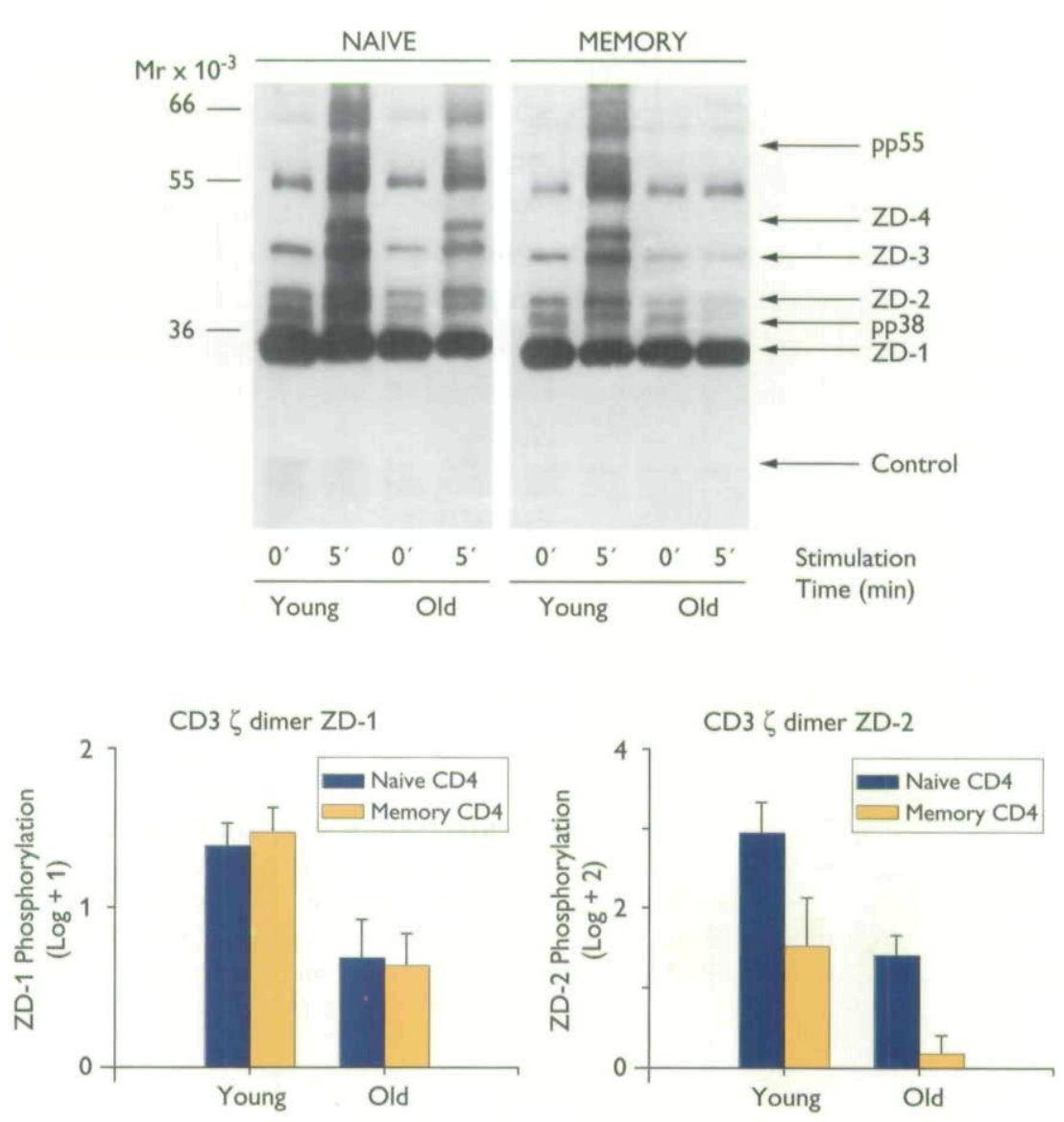

Fig. 4. Age- and subset-specific differences in phosphorylation of CD3 $\zeta$. The top panel shows an experiment in which naive (i.e. CD $44^{\mathrm{l}}$ ) and memory (i.e. $\mathrm{CD}_{4} 5 \mathrm{RB}^{\mathrm{lo}}$ ) $\mathrm{CD} 4 \mathrm{~T}$ cells were stimulated by anti-CD3/CD4 crosslinking for $5 \mathrm{~min}$ (or left unstimulated, in the $\mathrm{t}=0$ lanes); anti-CD3 $\zeta$ immunoprecipitates were then resolved by electrophoresis on non-reducing gels and detected by antiphosphotyrosine immunoblotting as in Fig. 3. The bottom panels show means and standard errors, after 5 min of activation, for a series of such experiments $(\mathrm{N}=4$ or 5 mice per point). Densitometric values were normalized as a ratio to a control band known not to vary with age, subset, or stimulation; log-transformed values are shown with an arbitrary constant added for graphical convenience. Statistical comparion described in the text. (G. Garcia \& R.A. Miller, unpublished data.) 
age and subset. CD3 $\zeta$ phosphorylation after 5 min of activation was higher in young naive than in young memory cells $(\mathrm{p}=$ 0.02 ) and higher in old naive than in old memory cells ( $p=$ 0.04); the difference was more than 10-fold at either age. There was also a 10 -fold effect of age within each subset $(\mathrm{p}=$ 0.03 for naive cells and $\mathrm{p}=0.06$ for memory cells). The responses of the other activation-sensitive $\mathrm{CD} 3 \zeta$ dimers $\mathrm{ZD}-3$ and ZD-4, as well as the $\zeta$-associated molecules p38 and p55, showed age- and subset-specific variations similar to that shown for ZD-2. Thus the accumulation of memory cells in aging mice contributes to the overall decline in CD3 $\zeta$ phosphorylation, but changes in the function of naive and memory cells also play a role.

Signal propagation: binding, phosphorylation, and activation of Zap-70

The decline with age in $\mathrm{CD} 3 \zeta$ phosphorylation suggested the hypothesis that aged T cells might also exhibit lower amounts of CD3-bound Zap-70, slower rates of Zap-70 activation by phosphorylation, and lower levels of Zap-70 kinase activity, either before or after activation. To test this idea, we generated $\mathrm{CD} 3 \zeta$ immunoprecipitates from resting $\mathrm{T}$ cells from old or young mice, and quantitated their Zap-70 levels by immunoblotting. The results (Fig. 5) showed an unexpected, but clear and consistent, increase in the amount of Zap-70 present in $\mathrm{CD} 3 \zeta$ immunoprecipitates from resting CD4 cells from aged donors. This increase in CD3-associated Zap-70 did not reflect a corresponding increase in the total amount of Zap-70, which remained essentially constant with age in whole cell lysates. The paradoxical increase with age in CD3 -associated Zap-70, despite the decline with age in $\mathrm{CD} 3 \zeta$ chain phosphorylation, suggests the hypothesis that the phosphorylated tyrosine residues on $\mathrm{CD} 3 \zeta$ chains of $\mathrm{T}$ cells from young mice, though more numerous than in old $\mathrm{T}$ cells, may be distributed in ways that are less conducive to Zap-70 association. Changes in the Zap-
70 molecule itself might also contribute to this age-related alteration in compartmentalization.

Does the increase with age in Zap-70 associated with $\mathrm{CD} 3 \zeta$ lead to a parallel increase in phosphorylation of the Zap-70 molecule, which might in turn lead to increases in Zap-70 kinase activity? To address the first of the these questions we used anti-PY immunoblotting to detect tyrosine-phosphorylated proteins in anti-CD3 $\zeta$ immunoprecipitates from resting CD4 cells and from CD4 cells 3 min after activation by anti-CD3 and anti-CD4 cross-linking. This method reveals a prominent PY phosphoprotein at MW $70 \mathrm{kDa}$, which we assume to be Zap-70. As shown in the top panel of Fig. 6, stimulation leads to only a small rise in Zap-70 phosphorylation, about $70 \%$ over baseline, in CD4 T cells from young mice. CD4 T cells from old mice do not show this increase, but this is because they have higher levels of Zap-70 phosphorylation in the resting state, and not because of any effect of age on the amount of phosphorylated Zap-70 after activation. Zap-70 protein kinase function, shown in the bottom panel of Fig. 6, shows a very similar pattern, with a significant stimulation seen only in young mice, and with an age-dependent increase in resting kinase function that produces similar levels of Zap-70 kinase activity in activated CD4 cells of young and old mice. Parallel studies of Jurkat and HT-2 cells showed that these transformed cell lines had both lower resting levels of Zap-70 kinase function than normal $\mathrm{T}$ cells, and higher kinase function after $3 \mathrm{~min}$ of stimulation, yielding a much larger stimulation index (not shown).

These data do not lend any support to the idea that alterations in Zap-70 binding to CD3 , Zap-70 phosphorylation, or Zap-70 kinase function contribute to age-related changes in T-cell activation. The test used in Fig. 6 to quantitate tyrosinespecific phosphorylation does not discriminate among the various phosphorylation sites on the Zap-70 molecule, some of which are thought to have a negative regulatory effect; our data thus do not exclude an effect of age on the distribution of phosphate groups within Zap-70. The in vitro kinase assay, based on
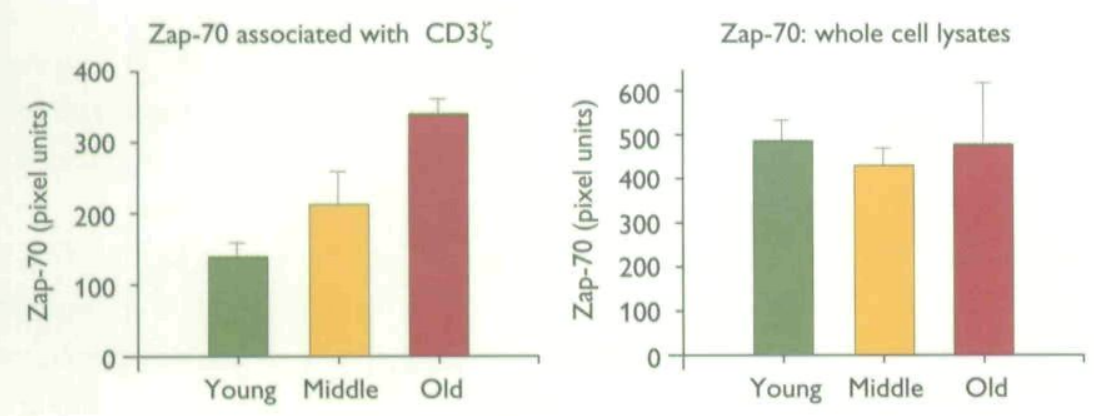

Fig. 5. Age-dependent increase in Zap-70 associated with the $\mathrm{CD} 3 \zeta$ chain. The left panel shows levels of Zap-70, detected by immunoblot in $\mathrm{CD} 3 \zeta$ immunoprecipitates as means \pm standard errors for 8 young, 6 middleaged, and 6 old mice. The right panel shows levels of Zap-70 in whole cell lysates from 6 young, 6 middle-aged, and 3 old mice. From G. Garcia \& R.A. Miller (submitted for publication). 


\section{Zap-70: tyrosine phosphorylation}

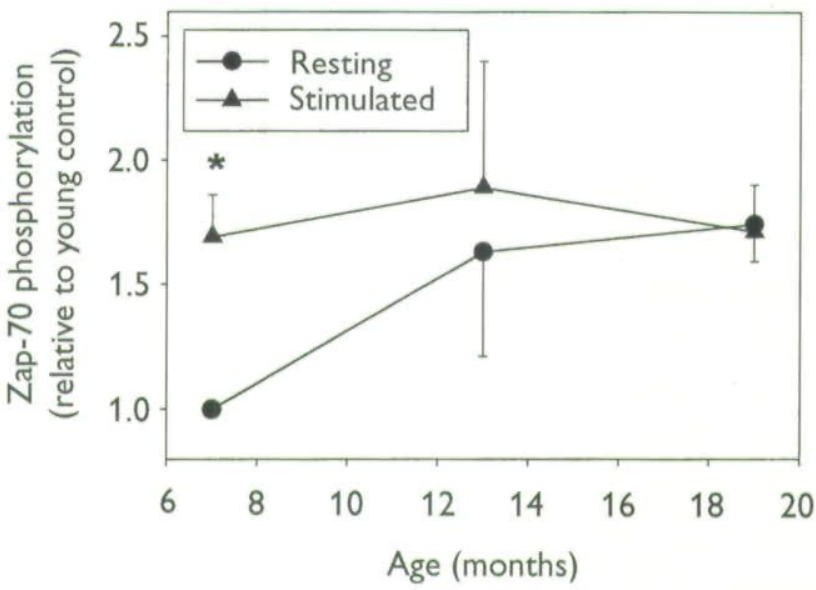

Zap-70: kinase function

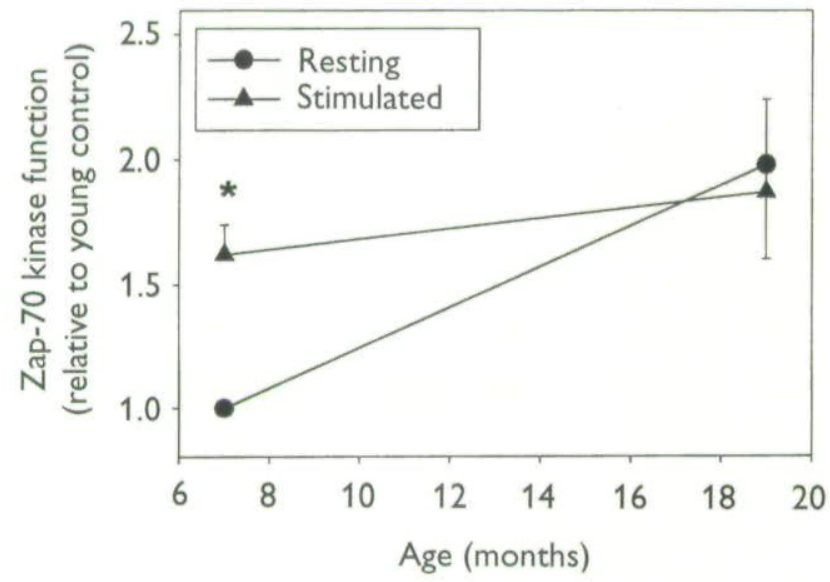

Fig. 6. Effects of age on Zap-70 tyrosine phosphorylation and kinase function. Top panel shows Zap-70 tyrosine phosphorylation using antiphosphotyrosine antibodies to detect phosphoproteins associated with $\mathrm{CD} 3 \zeta$ in immunoprecipitates of resting CD4 cells (circles) or cells stimulated for 3 min by cross-linking CD3 to CD4. Data are means ( \pm standard error) for 15 young, 10 middle-aged, and 10 old mice, expressing each value as a ratio to the unstimulated young cells tested in the same assay. Lower panel indicates tyrosine-specific kinase activity in an in vitro kinase assay using GST-Band III fusion protein as a Zap-70 substrate for tests of 5 young and 4 old mice. The asterisks indicate significant differences between resting and stimulated cells. From G. Garcia \& R.A. Miller (submitted for publication).

ability to transfer phosphate groups to a model substrate, erythrocyte Band III protein, might fail to detect subtle differences in Zap-70 function that could affect phosphorylation of T-cellspecific substrates attached to, or adjacent to, the CD3 complex. It is also quite possible that altered association of coupling or substrate proteins, or phosphatases, to $\mathrm{CD} 3 \zeta$, to Zap-70, or to other elements of the signal transduction complex might lead to a net decline in Zap-70 activity in situ in old T cells. Agerelated differences in association of Zap-70 to ITAM regions in the CD3 $\gamma \delta \varepsilon$ complex might also lead to functionally significant defects in T-cell activation. Lastly, it is possible that signal transduction defects in aged $\mathrm{T}$ cells are all distal to the CD3/Zap-70 complex. Clearly, additional studies will be needed to discriminate among these possibilities.

\section{Signal integration: Raf-1, MEK, ERK}

Investigations of Zap-70 kinase activity look at what is thought to be the earliest step in the activation cascade. The steps that immediately follow Zap-70 induction are still unclear and the topic of intense study in many laboratories. It seems likely that these steps involve, among other players, a variety of phospholipases and lipid kinases, proteins that activate Ras and its relatives, and several isoforms of PK-C, as well as sets of tyrosinespecific kinases and phosphatases. These interactions resolve themselves ultimately, i.e. within the first few minutes after signal initiation, into a number of final common pathways able to turn on new gene transcription to prepare the cell for entry into the cell cycle and production of cytokines and growth factor receptors. Among these key mediators of signal integration is the extracellular receptor-activated kinase ERK (also called MAP kinase). ERK function is initiated when the enzyme is phosphorylated on both tyrosine and threonine by the dual-specific kinase MEK, and MEK is itself regulated by the protein kinase Raf-1.

Fig. 7 shows results from a study (29) in which ERK function was measured in T cells from mice of various ages by its ability to phosphorylate a specific substrate, the ribosomal S6 protein kinase p90 $0^{\text {rsk }}$. T cells were stimulated by anti-CD3 cross-linking for 1-30 min, and the amount of phosphorylated p90 rsk monitored by anti-p90 immunoblotting; phosphorylated $\mathrm{p} 90^{\text {rsk }}$ can be discriminated from the unphosphorylated substrate by its diminished electrophoretic mobility. At the peak of the reaction, 10 min after T-cell stimulation, about $45 \%$ of the p $90^{\text {rsk }}$ had been converted by ERK to its slower migrating form in young cells, but only $30 \%$ in old T cells, as compared to a baseline of $5-7 \%$ in resting $\mathrm{T}$ cells. The differences between young and old $\mathrm{T}$ cells were statistically significant at this time point, and also at the 1, 2, and 5 min intervals (29).

Activation of ERK by MEK can be also be followed by an electrophoretic mobility shift technique, since phosphorylated ERK migrates more slowly than the unphosphorylated form. The bottom panel of Fig. 7 shows the results of a series 

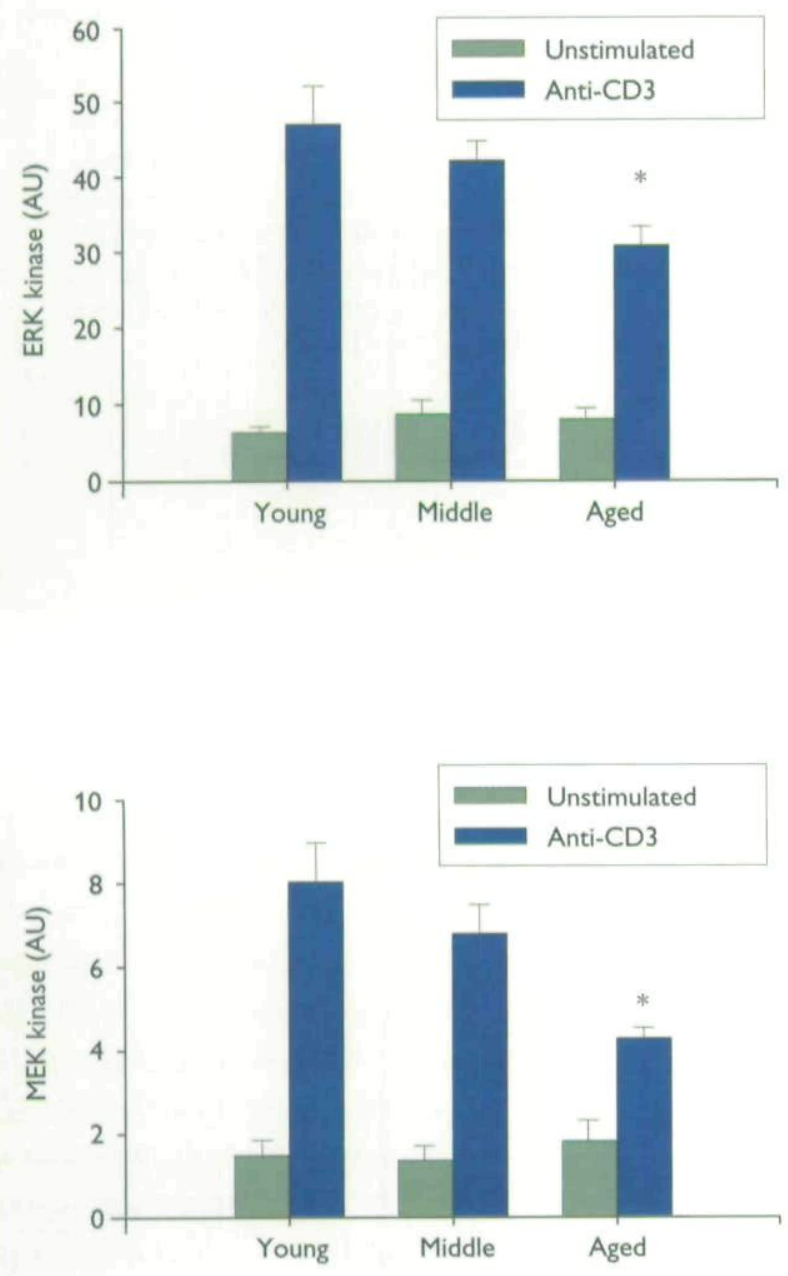

Fig. 7. Effects of age on ERK and MEK kinase function. Top panel shows ERK function, measured by conversion of $\mathrm{p} 90^{\text {rak }}$ to its phosphorylated form after 10 min exposure to anti-CD3 antibodies. Each bar shows mean \pm standard error for 6 mice; each $\mathrm{AU}$ (arbitrary unit) represents $1 \%$ conversion of $\mathrm{p} 90^{\text {rik }}$ to its slower migrating form. The asterisk indicates a significant difference between young and old mice. The bottom panel shows MEK function, measured by conversion of ERK2 to its slower migrating form after $2 \mathrm{~min}$ of activation by cross-linking CD3, as means \pm standard error for $\mathrm{N}=7$ experiments.

of assays of MEK function in T cells activated by cross-linked anti-CD3 antibodies. T cells from young mice contain about 8\% of their ERK in the phosphorylated state at the peak of the response ( 2 min after stimulation), compared to a baseline of $1 \%$ in resting $\mathrm{T}$ cells, but in old $\mathrm{T}$ cells only about $4 \%$ of the ERK is phosphorylated. This difference is statistically signifi-

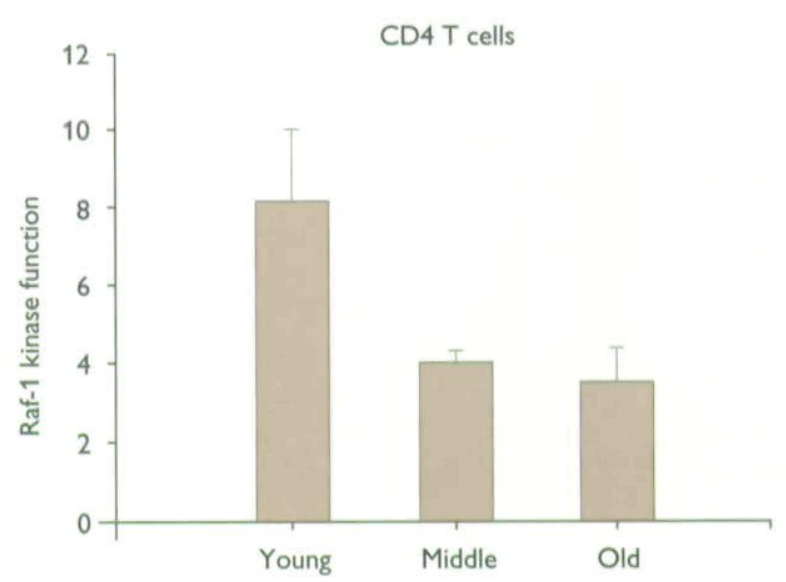

Subsets of CD4 pool

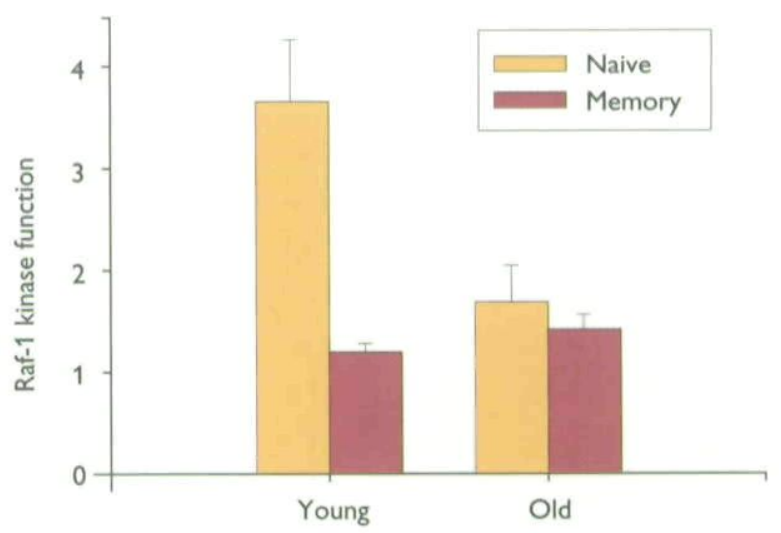

Fig. 8. Effects of age on Raf-1 kinase function in CD4 $\mathrm{T}$ cells and subsets. Top panel: CD4 T cells were stimulated for 5 min by crosslinking CD3 to CD4, and Raf-1 immunoprecipitates were used for an in vitro kinase assay employing a kinase-inactive version of MEK as substrate. Values show means and standard errors for 3 mice in each age group as the ratio of activity in stimulated cells to that in unstimulated cells; the young cells give significantly $(\mathrm{p}<0.05)$ higher values than the middleaged or old cells at this time point and at 2 and 10 min (not shown). Bottom panel: $\mathrm{CD} 4 \mathrm{~T}$ cells from young or old mice were separated into naive (i.e. $\mathrm{CD} 44^{\mathrm{l}}$ ) and memory (i.e. $\mathrm{CD} 62^{\mathrm{l}}$ ) subsets, which were then activated and assayed as in the top panel. Values are means and standard errors for $\mathrm{N}=5$ experiments. Young naive cells have significantly $(\mathrm{p}<$ 0.05 ) higher Raf-1 kinase function after activation than either young memory cells or old naive cells. From C.J. Kirk \& R.A. Miller (submitted).

cant in a series of 7 experiments. The data thus show an agedependent decline of about 2 -fold in peak activation levels of ERK, and suggest that an equivalent decline in activation of MEK can account for the ERK defect. Similar results have been reported for $\mathrm{T}$ cells from some, though not all, aged human donors (30). 

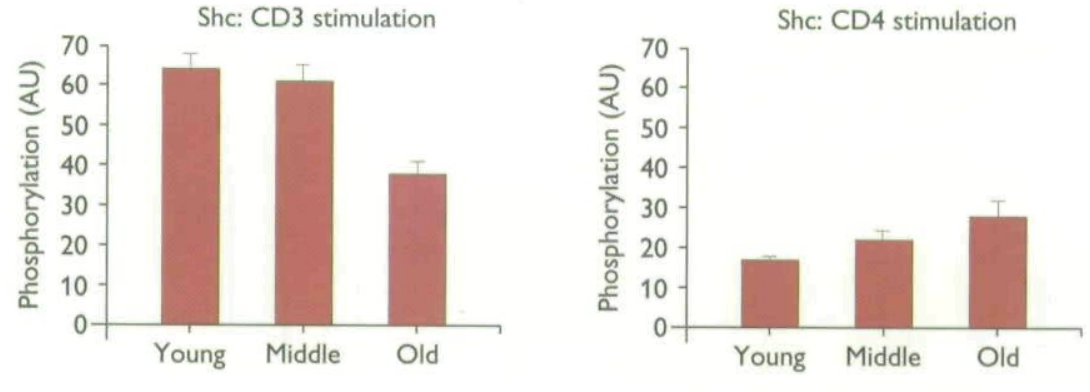

Fig. 9. Tyrosine phosphorylation of Shc in $\mathrm{T}$ cells: differential effects of anti-CD 3 and anti-CD4 stimulation. $\mathrm{T}$ cells from mice of different ages were stimulated either by crosslinking anti-CD3 (left panel) or anti-CD4 (right panel), and phosphorylated Shc detected using antiphosphotyrosine antibody. Bars show means and standard errors from $\mathrm{N}=4$ experiments. The effects of age are statistically significant $(\mathrm{p}<0.05)$ for both stimulators. From (31).
Since MEK can be activated by yet another protein kinase, Raf-1, we next carried out tests of Raf-1 function in young and old $\mathrm{T}$ cells, using $\mathrm{CD} 3 / \mathrm{CD} 4$ cross-linking as the stimulus. The results (Fig. 8) showed that CD4 T cells from young mice were substantially better at activating Raf- 1 than cells from middleaged or old donors. The decline with age does not reflect a parallel decline in the total amount of Raf-1 protein; in fact, the total amount of cellular Raf-1 increases significantly, about 2 fold, with age, and the decline in kinase specific activity (kinase function per mg of Raf-1 protein) is thus more severe than that suggested by Fig. 8 . The bottom portion of Fig. 8 shows that the effect of age can be attributed only in part to differences between naive and memory cells (in the CD4 set). Memory cells of young mice are substantially less responsive than naive cells to CD3/CD4 cross-linking in the Raf-1 kinase assay, and the increase with age in the proportion of memory cells will thus lead to a decline in overall Raf-1 inducibility. But aging also leads to a significant decline in function within the naive cell population. These results are formally similar to the subsetand age-dependent changes in $\mathrm{CD} 3 \zeta$-dimer phosphorylation shown in Fig. 4, but may well involve a different mechanism. In view of the clear-cut difference between young naive and young memory cells in Raf-1 activation shown in Fig. 8, it is puzzling that activation of MEK by anti-CD3 does not seem to differ between naive and memory CD4 cells of young mice (29). The discrepancy may involve differing stimuli - the Raf1 experiments used both CD3 and CD4 activation - or could reflect alternate pathways to MEK activation.

The demonstration of an age-associated decline in Raf-1 activation justifies a closer look at the macromolecules that regulate Raf-1 itself. At least two distinct pathways can induce Raf1 function in T cells, one involving PK-C phosphorylation of Raf- 1 , and the other requiring activation of Ras; the latter pathway also seems to require tyrosine-specific phosphorylation of Raf-1 by kinases unknown. Other enzymes related to MEK and ERK, including the c-jun N-terminal kinase JNK, may also be fruitful targets for further work.

\section{Altered circuitry: phosphorylation of Shc}

It is clear from work in many laboratories, as well as from some of our own findings presented above, that simple sequential models of T-cell activation do not adequately convey the complexity of the signal transduction process, which must include an elaborate array of feedback loops and qualitative modulations, some of them possibly subset-specific, others invoked only in the presence of particular co-stimulators or by agonists whose affinity for the TCR falls within a particular range. Some insight into the way in which aging might alter this circuitry has emerged from a study of tyrosine phosphorylation of the Shc protein (31). In many cell types Shc helps to couple transmission of signals from a tyrosine-phosphorylated substrate (such as phosphorylated $\mathrm{CD} 3 \zeta$ ) to Ras by promoting the accumulation at the transduction complex of the Ras-activating Grb2/mSOS heterodimer. The question of whether or not Shc plays such a role in T cells is controversial $(32,33)$, but there is general agreement that Shc can serve as a substrate for one or more of the tyrosine-specific protein kinases activated in the early phases of T-cell activation. We have examined the extent of Shc tyrosine phosphorylation in T cells after activation by two routes (Fig. 9). In responses to anti-CD3 cross-linking, T cells from aged mice generated about half as much phosphorylated Shc as did T cells from young donors; this result is consistent with the data presented above on MEK, ERK, and Raf-1 function. In contrast, when the cells were stimulated by crosslinking $\mathrm{CD} 4$, in the absence of any anti-CD3 signal, then old T cells generated almost 2 -fold more Shc phosphorylation than did young T cells. This result suggests that the factors that regulate activation of the CD4-associated kinase lck - its association with $\mathrm{CD} 4$, its own phosphorylation state, its location within the cell membrane - may be altered by aging in ways that interfere with lck triggering after $\mathrm{CD} 3$ clustering but facilitate lck function when CD4 molecules are themselves aggregated by anti-CD4 antibody. There is evidence that CD3 and CD4 (together with the tyrosine-protein phosphatase CD45) 
may be in close proximity on memory $\mathrm{T}$ cells, but not in naive T cells (34), and it will be important to determine whether the age-related increase in Shc phosphorylation induced by antiCD4 represents differences between naive and memory T cells, an effect of aging within these subsets, or a combination of both effects.

\section{Concluding remarks}

Researchers interested in the ways in which aging alters the T-cell activation process have so far glimpsed only a few aspects of the proverbial elephant - or perhaps several different elephants, one for each of the various T-cell subsets. The picture that is emerging is not the simplest one, in which T-cell signals stumble at one well-defined early step, with all subsequent steps choked off proportionally. Instead, aging seems to alter the signalling network in subtler ways, with some specific mediators (or reactions) set at higher levels, and others at lower levels, and with some variations that appear to be specific for individual T-cell subsets. Growing appreciation of the detailed mechanisms of signal transduction in T-cell lymphomas and, even more helpfully, in freshly isolated resting $\mathrm{T}$ cells and their subsets will in coming years facilitate the dissection of the biochemistry of T-cell immune senescence. Studies of the responses of aged TCR-transgenic mice to defined peptide antigens (or partial agonists) may also provide important clues. Nonetheless, drawing convincing inferences about the functional implications of these biochemical changes is likely to remain a formidable challenge for some time.

\section{References}

1. Wange RL, Samelson LE. Complex complexes: signaling at the TCR. Immunity 1996;5:197-205.

2. Chan AC, Desai DM, Weiss A. The role of protein tyrosine kinases and protein tyrosine phosphatases in $\mathrm{T}$ cell antigen receptor signal transduction.

Annu Rev Immunol 1994; 12:555-592.

3. Philosophe B, Miller RA. Diminished calcium signal generation in subsets of $\mathrm{T}$ lymphocytes that predominate in old mice.

J Gerontol A Biol Sci Med Sci 1990;45: B87-B93.

4. Rajasekar R, Augustin A. Antigen-dependent selection of $\mathrm{T}$ cells that are able to efficiently regulate free cytoplasmic calcium levels. J Immunol 1994;153:1037-1045.

5. Grossmann A, Ledbetter JA, Rabinovitch PS. Aging-related deficiency in intracellular calcium response to anti-CD3 or concanavalin A in murine T-cell subsets.

J Gerontol A Biol Sci Med Sci 1990;45: B81-B86.

6. Miller RA. Calcium signals in $\mathrm{T}$ lymphocytes from old mice.

Life Sci 1996:59:469-475.

7. Miller RA, Jacobson B, Weil G, Simons ER, Diminished calcium influx in lectinstimulated T cells from old mice. J Cell Physiol 1987; 132:337-342.

8. Miller RA, Philosophe B, Ginis I, Weil G, Jacobson B. Defective control of cytoplasmic calcium concentration in $\mathrm{T}$ lymphocytes from old mice.

J Cell Physiol 1989;138:175-182.
9. Negoro $\mathrm{S}, \mathrm{Hara} \mathrm{H}$. The effect of taurine on the age-related decline of the immune response in mice: the restorative effect on the $\mathrm{T}$ cell proliferative response to costimulation with ionomycin and phorbol myristate acetate. Adv Exp Med Biol 1992;315:229-239.

10. Lerner A, Philosophe B, Miller RA. Defective calcium influx and preserved inositol phosphate generation in $\mathrm{T}$ cells from old mice.

Aging Immunol Infect Dis 1988;1:149-157.

11. Gelfand EW, Cheung RT, Mills GB, Grinstein S. Uptake of extracellular $\mathrm{Ca}^{2+}$ and not recruitment from internal stores is essential for $\mathrm{T}$ lymphocyte proliferation. Eur J Immunol 1988;18:917-922.

12. Philosophe B, Miller RA. T lymphocyte heterogeneity in old and young mice: functional defects in $\mathrm{T}$ cells selected for poor calcium signal generation. Eur J Immunol 1989;19:695-699.

13. Miller RA, Flurkey K, Molloy M, Luby T, Stadecker MJ. Differential sensitivity of virgin and memory $\mathrm{T}$ lymphocytes to calcium ionophores suggests a bouyant density separation method and a model for memory cell hyporesponsiveness to $\mathrm{Con} \mathrm{A}$. J Immunol 1991;147:3080-3086.

14. Witkowski JM, Miller RA. Increased function of P-glycoprotein in $\mathrm{T}$ lymphocytes of aging mice.

J Immunol 1993;150:1296-1306.

15. Bining N, Miller RA. Cytokine production by subsets of $\mathrm{CD} 4$ memory $\mathrm{T}$ cells differing in P-glycoprotein expression: effects of aging. J Gerontol A Biol Sci Med Sci 1997; 52A: B137-B145.
16. Linton PJ, Haynes L, Klinman NR, Swain SL. Antigen-independent changes in naive CD4 $\mathrm{T}$ cells with aging. J Exp Med 1996;184:1891-1900.

17. Patel HR, Miller RA. Analysis of protein phosphorylation patterns reveals unanticipated complexity in $\mathrm{T}$ lymphocyte activation pathways. J Immunol 1991;146:3332-3339.

18. Patel HR, Miller RA. Age-associated changes in mitogen-induced protein phosphorylation in murine $T$ lymphocytes. Eur J Immunol 1992;22:253-260.

19. Fulop T Jr., Leblanc C, Lacombe G, Dupuis G. Cellular distribution of protein kinase $\mathrm{C}$ isozymes in CD3-mediated stimulation of human $\mathrm{T}$ lymphocytes with aging. FEBS Lett 1995; 375:69-74.

20. Monks CR, Kupfer H, Tamir I, Barlow A, Kupfer A. Selective modulation of protein kinase $\mathrm{C}$-theta during $\mathrm{T}$-cell activation. Nature 1997; 385:83-86.

21. Shi J, Miller RA. Tyrosine-specific protein phosphorylation in response to anti- $\mathrm{CD} 3$ antibody is diminished in old mice. J Gerontol A Biol Sci Med Sci 1992;47: B147-B153.

22. Shi J, Miller RA. Differential tyrosine-specific protein phosphorylation in mouse T lymphocyte subsets. Effect of age. J Immunol 1993;151:730-739.

23. Chan $A C$, Irving $B A$, Fraser JD, Weiss $A$. The zeta chain is associated with a tyrosine kinase and upon T-cell antigen receptor stimulation associates with ZAP-70, a 70-kDa tyrosine phosphoprotein. Proc Natl Acad Sci USA 1991;88:9166-9170. 
24. van Oers NS, Killeen N, Weiss A. ZAP-70 is constitutively associated with tyrosinephosphorylated TCR zeta in murine thymocytes and lymph node T cells. Immunity 1994;1:675-685.

25. Garcia GG, Miller RA. Differential tyrosine phosphorylation of zeta chain dimers in mouse CD4 T lymphocytes: effect of age. Cell Immunol 1997;175:51-57.

26. Sancho J, et al. Coupling of GTP-binding to the $\mathrm{T}$ cell receptor (TCR) zeta-chain with TCR-mediated signal transduction. J Immunol 1993;150:3230-3242.

27. van Oers NS, Killeen N, Weiss A. Lck regulates the tyrosine phosphorylation of the $\mathrm{T}$ cell receptor subunits and $\mathrm{ZAP}-70$ in murine thymocytes.

J Exp Med 1996;183:1053-1062.
28. Miller RA. Aging and the immune response. In: Schneider EL, Rowe JW, eds. Handbook of the biology of aging. San Diego, CA: Academic Press; 1996. p.355-392.

29. Gorgas G, Butch ER, Guan KI, Miller RA. Diminished activation of the MAP kinase pathway in CD3-stimulated T lymphocytes from old mice. Mech Ageing Dev 1997;91:74-83.

30. Whisler RL, Newhouse YG, Bagenstose SE. Age-related reductions in the activation of mitogen-activated protein kinases p44mapk/ERK1 and p42 mapk/ERK2 in human $T$ cells stimulated via ligation of the $T$ cell receptor complex.

Cell Immunol 1996;168:201-210.
31. Ghosh J, Miller RA. Rapid tyrosine phosphorylation of Grb2 and Shc in T cells exposed to anti-CD3, anti-CD4, and antiCD45 stimuli: differential effects of aging. Mech Ageing Dev 1995;80:171-187.

32. Osman N, Lucas SC, Turner H, Cantrell D. A comparison of the interaction of Shc and the tyrosine kinase ZAP-70 with the T cell antigen receptor zeta chain tyrosine-based activation motif.

J Biol Chem 1995;270:13981-13986.

33. Ravichandran KS, Lorenz U, Shoelson SE, Burakoff SJ. Interaction of Shc with Grb2 regulates association of Grb2 with mSOS. Mol Cell Biol 1995; 15:593-600.

34. Dianzani U, et al. Molecular associations on the $T$ cell surface correlate with immunological memory. Eur J Immunol 1990;20:2249-2257. 
This document is a scanned copy of a printed document. No warranty is given about the accuracy of the copy. Users should refer to the original published version of the material. 